\title{
What Is Your Diagnosis?
}

In collaboration with the American College of Veterinary Radiology
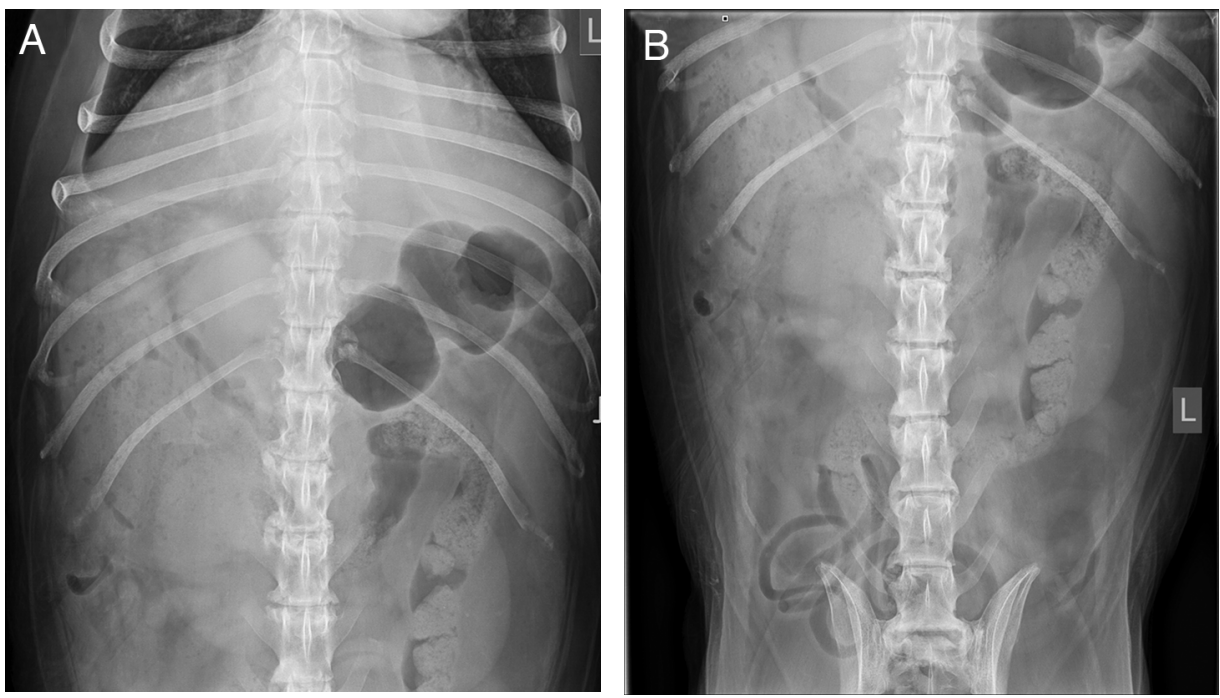

Figure I-Ventrodorsal cranial (A) and caudal (B) and left lateral cranial (C) and caudal (D) abdominal radiographic images of an II-year-old spayed female Golden Retriever evaluated because of icterus, anorexia, and weakness.
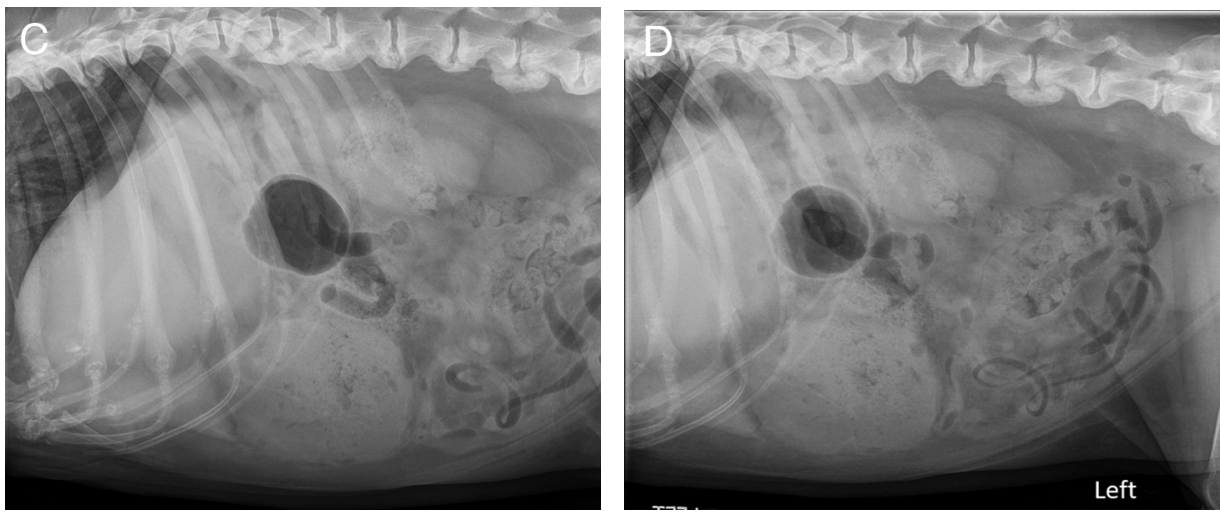

\section{History}

An 11-year-old spayed female Golden Retriever was referred for icterus and 2 days of anorexia. On clinical examination, the patient was icteric, best

Carlo Anselmi, DVM ${ }^{*}$; Kaiwen Chen, BVSc ${ }^{2}$; Hannah Turner, DVM ${ }^{3}$; David Biller, DVM ${ }^{3}$; Kara Berke, DVM ${ }^{3}$; Nicolette Cassel, BVSc ${ }^{3}$

'Pride Veterinary Centre, Derby, UK

${ }^{2}$ College of Veterinary Medicine, Kansas State University, Manhat$\tan , \mathrm{KS}$

${ }^{3}$ Department of Clinical Sciences, College of Veterinary Medicine, Kansas State University, Manhattan, KS

*Corresponding author: Dr. Anselmi (anselmi.carlo@gmail.com)

https://doi.org//0.2460/javma.20.II.062I demonstrated on the sclera, ventral abdomen, pinnae, and mucous membranes. The patient was weak but without neurologic deficits. All vital signs were within reference limits. Results of $\mathrm{CBC}$ and serum biochemical analyses indicated anemia (Hct, 29\%; reference range, $41 \%$ to $59 \%$ ), band neutrophil and monocytosis, high activities of alanine transaminase $(4,018 \mathrm{U} / \mathrm{L}$; reference range, 20 to $144 \mathrm{U} / \mathrm{L}$ ) and alkaline phosphatase (2,557 U/L; reference range, 10 to $130 \mathrm{U} / \mathrm{L})$, and high total bilirubin concentration $(9.4 \mathrm{mg} / \mathrm{dL}$; reference range, 0.0 to $0.2 \mathrm{mg} / \mathrm{dL}$ ). No previous surgery, trauma, or systemic illness was reported. Abdominal radiography was performed (Figure I).

Formulate differential diagnoses, then continue reading. 


\section{Diagnostic Imaging Findings and Interpretation}

Abdominal radiography revealed an irregularly marginated soft tissue opaque mass with stippled to coalescing gas bubbles, creating a vesicular-like gas pattern in the right ventral aspect of the abdomen immediately caudal to the liver and ventral to the pylorus (Figure 2). On lateral and ventrodorsal projections, the mass was partially confluent with the hepatic silhouette. There was adjacent decreased serosal detail, with wispy soft tissue opacity present within the ventral and right abdomen. Multifocal type 3 spondylosis deformans was present, with lateral extension of bone formation as well as moderate spondyloarthropathy of articular processes of L4 and L5. The dorsal aspect of the spleen (head of the spleen) was considered to have been in a normal anatomic position, without radiographic signs of torsion. The other abdominal structures were unremarkable. Organs of origin of the mass included liver or, less likely, spleen; a pancreatic origin could not be completely excluded. Radiographic differential diagnoses for the emphysematous mass included the presence of an abscess, necrotic-suppurative neoplasm, or other ischemic-necrotic process; a concurrent liver lobe torsion was also considered.

Abdominal ultrasonography (Figure 3) was performed and revealed a heterogeneously hypoechoic

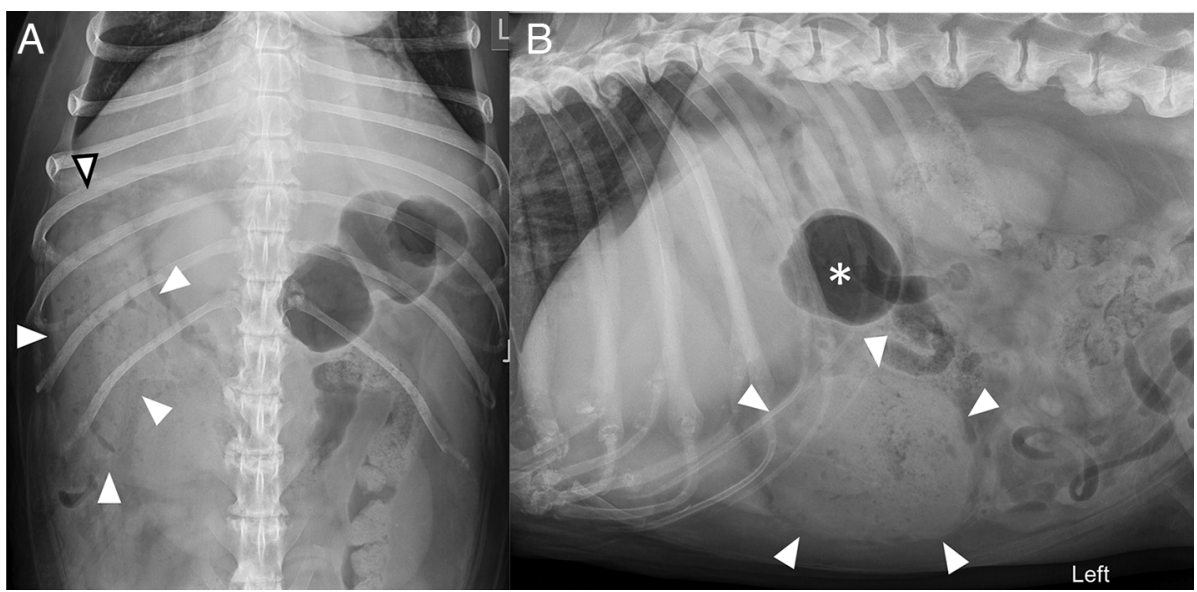

Figure 2-Same cranial abdominal radiographic images as in Figure I. A-There is an irregularly marginated soft tissue opacity mass (arrowheads) with stippled to coalescing gas bubbles at the right and ventral aspect of the cranial abdomen, partially confluent (white arrowhead with black border) with the hepatic silhouette. B-The mass (arrowheads) is caudal and ventral to the liver, partially silhouetting with the hepatic margins and dorsally displacing the pylorus (white asterisk). Adjacent to the mass, there is focal decreased serosal detail with wispy soft tissues present within the ventral and right abdomen. Diffuse type 3 spondylosis deformans is also present affecting multiple contiguous vertebrae as well as moderate spondyloarthropathy of the articular processes of L4 and L5.
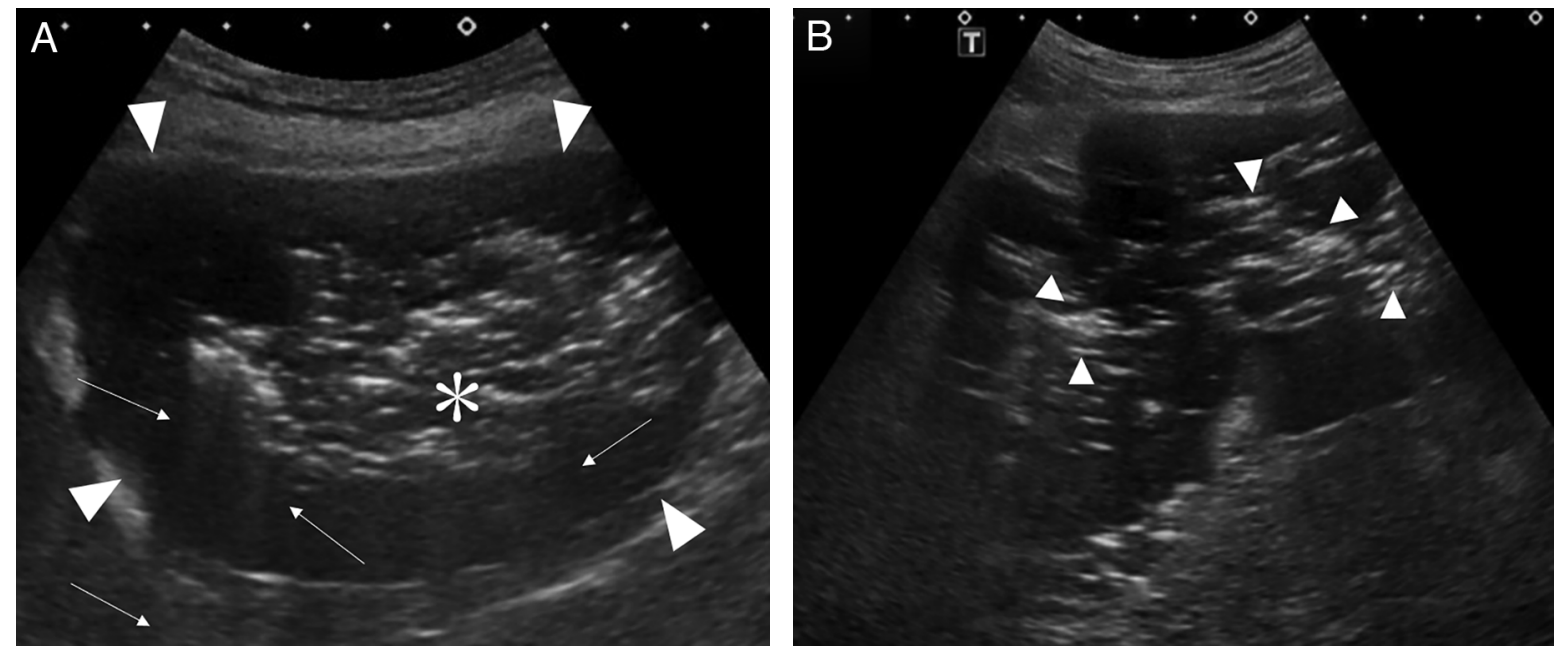

Figure 3-Parasagittal (A) and transverse (B) plane ultrasonographic images of the right cranial abdomen of the same dog. A-There is a well-defined, irregularly marginated, large hypoechoic mass lesion (arrowheads) caudal and contiguous to the right side of the liver. It is delimitated by a hypoechoic band with a central accumulation of hyperechoic areas (asterisk) characterized by multiple reverberation artifacts (arrows), consistent with gas. B-Notice the lacy pattern with gas accumulating in a similar fashion within the mass (arrowhead). 
mass with coarse echotexture and multiple interspersed linear echoes giving a lacy pattern appearance. No vascular flow was noted on color flow Doppler interrogation. Reverberation artifacts were diffusely present within the mass, indicating the presence of intralesional gas. The mass was delimitated by a thick hypoechoic band and extended from the caudal margin of the right hepatic lobe. A normal caudate lobe of the liver could not be visualized in the expected position cranial to the right kidney. A minimal amount of hypoechoic abdominal free fluid was detected. The other abdominal structures, including the spleen, were unremarkable. Ultrasonographic differential diagnoses for the abnormal findings included caudate or right lateral liver lobe torsion with concurrent parenchymal hepatic emphysema, hepatic abscess formation, acute infarction, or neoplastic necrotic or suppurative process. Secondary peritonitis was considered the most likely differential diagnosis for the abdominal effusion.

\section{Treatment and Outcome}

Emergency exploratory laparotomy was performed. At surgery, torsion of the right lateral liver lobe and a large, cavitated mass with pungent odor were found as well as minimal abdominal effusion. Right lateral liver lobectomy was performed, and the removed tissue was submitted for histology. Samples of the abdominal effusion were submitted for aerobic and anaerobic bacterial culture. The patient recovered uneventfully.

Histopathology confirmed acute, diffuse necrosis of the liver lobe with no evidence of neoplasia or infection. The changes were consistent with ischemic damage associated with hepatic lobe torsion. Results were negative for the abdominal fluid bacterial cultures. Three weeks after the surgery, the patient was presented for follow-up examination, and no abnormalities were noted on physical examination.

\section{Comments}

Hepatic emphysema is an umbrella term to describe radiographic presence of gas within the liver parenchyma, portal veins, or biliary tract, alone or in combination. When gas is identified in the liver, it is pertinent from a diagnostic and prognostic aspect to distinguish in which component it is located. Intraparenchymal gas location can be secondary to a plethora of causes such as infection, sepsis, trauma, neoplasia, liver lobe torsion, infarct, and hepatic abscess formation. The presence of gas within the portal vasculature can be secondary to systemic or abdominal disease. Gas within the biliary system can be associated with duodenal gas reflux or infectious disease. Additionally, the presence of gas in any of the hepatic components can be secondary to iatrogenic causes. ${ }^{1}$
Radiographic differential diagnoses for hepatic gas accumulation vary depending on its pattern of distribution and localization (diffuse vs focal) and pattern (branching, solitary, coalescing, or other patterns). Branching and linear gas opacities in the liver indicate the accumulation of gas in the biliary system or vasculature, which was not appreciated in this case. In our case, the gas foci were more oval, coalescing with a vesicular-like gas pattern, and focally distributed to the right side and ventrally in the abdominal cavity, partially confluent with the liver. Therefore, we listed abscess formation as the primary radiographic differential diagnosis, with the liver as the likely organ of origin. In radiology, a vesicular gas pattern has been historically associated with lung lobe torsion and recently described also in necrotizing pneumonia. ${ }^{2}$ More recently vesicular-like gas pattern has been introduced as a term to describe the radiographic presence of gas in the spleen in spontaneous emphysematous splenitis in dogs. ${ }^{3}$ Due to similar radiographic appearance, we also used this term to describe this peculiar gas pattern identified in our patient.

Ultrasonography has been recently described as an important tool to differentiate the location of gas in the liver. ${ }^{1}$ In agreement with this, the presence of reverberation artifacts within the liver on ultrasonography confirmed the finding of hepatic parenchymal emphysema. Moreover, abdominal ultrasonography was integral for the concurrent diagnosis of liver lobe torsion. Previous literature describes ultrasonographic changes associated with splenic torsion as a lacy pattern of the spleen, characterized by a coarse, heterogeneous hypoechoic parenchyma and absence of blood flow on color flow Doppler interrogation. ${ }^{4}$ A hypoechoic band has also been recently described as an ultrasonographic feature of lung lobe torsion in dogs. ${ }^{2}$ Similarly, in our patient a hypoechoic thick band was visible delineating the gaseous hepatic lesion. The lacy pattern, together with the loss of the expected anatomic ultrasonographic landmarks (eg, renal fossa and right kidney) and the absence of blood flow in the right lateral or caudate liver lobe of this patient, increased the suspicion of lobe torsion. The presence of the hypoechoic band was an interesting finding, but a definitive explanation is beyond the scope of this report, and further investigations are needed to associate this feature with hepatic lobe torsion.

Hepatic lobe torsion commonly affects the left lateral lobe less than other lobes. Possible causes are ligamentous support pathology (congenital or acquired traumatic), neoplastic process, lobar enlargement, sufficient abdominal effusion, diaphragmatic hernia, as well as an idiopathic process. ${ }^{5,6}$ For the dog of the present report, there was minimal abdominal effusion, the presence of which could have been secondary to the liver lobe torsion rather than the cause. No history of trauma was reported in our patient, so this etiology was not included as a possible cause of either torsion or hepatic emphysema. 
The presence of hepatic parenchymal emphysema may be a negative prognostic factor, as it can indicate necrosis or concomitant infection. ${ }^{1}$ In our patient, hepatic infection due to gas-forming anaerobic bacteria (namely Clostridium spp) was suspected during the diagnostic imaging studies and surgery, as this was previously described for liver lobe torsion.? Interestingly, an infectious process was not definitely supported by the laboratory results for the dog of the present report. The absence of bacteria in histopathology could have been a false-negative result, possibly related to the presence of a large amount of necrotic and degenerated material from the lesion as well as because bacterial culture of hepatic tissue was not performed. Even with a hepatic infection, abdominal effusion may be sterile due to the absence of hepatic lobe rupture. The final diagnosis of hepatic necrosis of the resected lobe could have been explained by vascular compromise secondary to liver lobe torsion leading to focal ischemic processes. Excluding an infectious cause, the hepatic gas accumulation visible on radiography and ultrasonography could have been secondary to the torsion, due to the release of intracellular gas (oxyhemoglobin) from the necrotic tissue, as previously described. ${ }^{4}$ Alternatively, another explanation could have been a primary hepatic gas accumulation causing liver lobe enlargement leading to secondary lobar torsion. However, this temporal sequence of events was considered less likely because the focal lobar presence of gas would be difficult to otherwise explain. In fact, in this scenario, the gas would be possibly expected to diffuse to more lobes or more organs instead of accumulating only in a single hepatic lobe, as in this case. It was likely that the primary vascular occlusion in the dog of the present report functioned as a barrier, avoiding the spread of the gas. It was, therefore, speculated that in this patient, the acute torsion of the right lateral liver lobe possibly caused acute localized passive hepatic congestion, arterial and venous thrombosis, and eventually ischemic necrosis with gas accumulation. Iatrogenic causes were not considered as this was not supported by the clinical history of our patient.
Abdominal radiography was important to detect a space-occupying lesion with an associated vesicularlike gas pattern, and ultrasonography was beneficial to effectively confirm the intralesional gas and origin of the mass. In addition, the presence of a lacy pattern and absence of vascularization, along with the loss of expected anatomic landmarks, was ancillary in the concurrent diagnosis of liver lobe torsion. Even if uncommon, liver lobe torsion with necrotic hepatic emphysema has to be considered as a differential diagnosis in dogs with similar radiographic and ultrasonographic findings.

\section{Acknowledgments}

The authors declare that there were no financial or other conflicts of interest. Dr. Chen was sponsored by the China Scholarship Council and US-China Center for Animal Health.

Dr. Chen was a fourth-year veterinary student at the College of Veterinary Medicine, Kansas State University, Manhattan, KS when the report was written.

\section{Reference}

1. Manfredi S, Fabbi M, Bonazzi M, et al. Ultrasonographic differentiation between portal venous and parenchymal gas may be important for the prognosis of canine and feline hepatic emphysema: 37 cases. Vet Radiol Ultrasound. 2019;60:734-744.

2. Belmudes A, Gory G, Cauvin E, et al. Lung lobe torsion in 15 dogs: peripheral band sign on ultrasound. Vet Radiol Ultrasound. 2020;62(1):116-125.

3. Battiato P, Fernandez Y, Salgüero R, et al. Spontaneous emphysematous splenitis: radiographic and ultrasonographic findings in three Golden Retriever dogs. Vet Radiol Ultrasound. 2020;62(3):309-315.

4. Schelling CG, Wortman JA, Saunders HM. Ultrasonic detection of splenic necrosis in the dog: three case reports of splenic necrosis secondary to infarction. Vet Radiol Ultrasound. 1988;29:227-233.

5. Sonnenfield JM, Armbrust LJ, Radlinsky MA, et al. Radiographic and ultrasonographic findings of liver lobe torsion in a dog. Vet Radiol Ultrasound. 2001;42:344-346.

6. Swann HM, Brown DC. Hepatic lobe torsion in 3 dogs and a cat. Vet Surg. 2001;30:482-486.

7. Sato AF, Solano M. Radiographic diagnosis: liver lobe entrapment and associated emphysematous hepatitis. Vet Radiol Ultrasound. 1998;39:123-124. 